\title{
L'Intérêt et les limites de la recherche sur l'art de la miniature au Canada. La collection de Bibliothèque et Archives Canada
}

\section{Anne-Elisabeth Vallée}

Volume 30, numéro 1-2, 2005

The Portrait Issue

La question du portrait

URI : https://id.erudit.org/iderudit/1069663ar

DOI : https://doi.org/10.7202/1069663ar

Aller au sommaire du numéro

\section{Éditeur(s)}

UAAC-AAUC (University Art Association of Canada | Association d'art des universités du Canada)

ISSN

0315-9906 (imprimé)

1918-4778 (numérique)

Découvrir la revue

Citer cet article

Vallée, A.-E. (2005). L'Intérêt et les limites de la recherche sur l'art de la miniature au Canada. La collection de Bibliothèque et Archives Canada. RACAR : Revue d'art canadienne / Canadian Art Review, 30(1-2), 59-73. https://doi.org/10.7202/1069663ar

\section{Résumé de l'article}

While rediscovering Library and Archives Canada's collection of Canadian miniature portraits, this article wishes to shed light on the difficulties of research on miniature painting in Canada. The practice of miniature painting appeared in Canada shortly after the British Conquest of 1763, and became one of the most accessible ways to obtain a "likeness" before the invention of photography about a century later. An examination of LAC's collection shows the problems that originate from the loss of important information about the miniatures, such as the identity of the model and the artist, the production date and site. This loss of information is partly due to the itinerant way of life of many professional miniaturists, whose identity was lost over the years. To be able to stay in a city for a longer period, professional miniaturists offered their customers a diversified array of services, therefore producing fewer miniatures. As shown by some of the works in LAC's collection, a high quality miniature is not necessarily the work of a professional miniaturist, but may be the work of an amateur. Despite the loss of essential information, art historians manage to date the works within their approximate period by analysing different components such as the scale, the medium, and the subject's clothing. In fact, the development of miniature painting in Canada follows roughly the same stages as in France, England, and the United States. From the early very small-scale oval portraits painted in watercolour on ivory, miniature portraits evolved to resemble large-scale portraits and photographs. During the revival of the miniature portrait at the end of the nineteenth century, miniaturists returned to the specificities of the art, by, for example, working on a small scale and using jewel-like frames. Another interesting avenue of research on miniature portraits lies in the different roles played by the miniature in the private or social life of the owners. Always an object of memory, the miniature may be worn as an ornament, or, because of its high symbolic value, may become a fetish.
Tous droits réservés (c) UAAC-AAUC (University Art Association of Canada | Association d'art des universités du Canada), 2006
Ce document est protégé par la loi sur le droit d'auteur. L'utilisation des services d'Érudit (y compris la reproduction) est assujettie à sa politique d'utilisation que vous pouvez consulter en ligne.

https://apropos.erudit.org/fr/usagers/politique-dutilisation/ 


\title{
L'Intérêt et les limites de la recherche sur l'art de la miniature au Canada. La collection de Bibliothèque et Archives Canada
}

\author{
ANNE-ELISABETH VALLÉE, UNIVERSITÉ DU QuÉBEC A Montréal
}

\begin{abstract}
While rediscovering Library and Archives Canada's collection of Canadian miniature portraits, this article wishes to shed light on the difficulties of research on miniature painting in Canada. The practice of miniature painting appeared in Canada shortly after the British Conquest of 1763, and became one of the most accessible ways to obtain a "likeness" before the invention of photography about a century later. An examination of LAC's collection shows the problems that originate from the loss of important information about the miniatures, such as the identity of the model and the artist, the production date and site. This loss of information is partly due to the itinerant way of life of many professional miniaturists, whose identity was lost over the years. To be able to stay in a city for a longer period, professional miniaturists offered their customers a diversified array of services, therefore producing fewer miniatures. As shown by some of the works in LAC's collection, a high quality miniature is not necessarily the work of a professional miniaturist, but may be the
\end{abstract}

work of an amateur. Despite the loss of essential information, art historians manage to date the works within their approximate period by analysing different components such as the scale, the medium, and the subject's clothing. In fact, the development of miniature painting in Canada follows roughly the same stages as in France, England, and the United States. From the early very small-scale oval portraits painted in watercolour on ivory, miniature portraits evolved to resemble large-scale portraits and photographs. During the revival of the miniature portrait at the end of the nineteenth century, miniaturists returned to the specificities of the art, by, for example, working on a small scale and using jewel-like frames. Another interesting avenue of research on miniature portraits lies in the different roles played by the miniature in the private or social life of the owners. Always an object of memory, the miniature may be worn as an ornament, or, because of its high symbolic value, may become a fetish.
À

l'occasion de l'ouverture prochaine du Musée du portrait du Canada à Ottawa, nous nous proposons dans ce court article de traiter de la recherche concernant une modalité particulière de l'art du portrait, la miniature'. Cette pratique artistique, qui fait son apparition au Canada à la suite de la Conquête britannique, demeure, jusqu'à l'invention de la photographie un siècle plus tard, l'un des moyens les plus accessibles de produire la "ressemblance ${ }^{2}$ ". Cependant, bien avant l'arrivée des troupes britanniques, les colons français connaissaient déjà cette technique qui leur permettait de conserver les portraits de leurs proches restés sur le vieux continent. Même si les historiens d'art canadiens se préoccupent depuis longtemps de cette forme d'art, le premier ouvrage entièrement consacré à la miniature canadienne ne paraît qu'en 1981, année du dépôt du mémoire de Roslyn M. Rosenfeld intitulé Miniatures and Silhouettes in Montreal, 1760-18603. Avant cette date, deux précurseurs avaient néanmoins tracé le chemin en s'intéressant aux artistes actifs au Canada durant les XVIII et $\mathrm{XIX}^{\mathrm{e}}$ sic̀cles, et en particulier à certains miniaturistes. D'abord, Gérard Morisset avait publié, outre certains articles ${ }^{4}$, l'ouvrage La Peinture traditionnelle au Canada français qui retrace l'histoire de l'art au Québec depuis ses origines jusqu'au début du XX $\mathrm{XX}^{\mathrm{c}}$ siècle, projet ambitieux qui aborde le cas de quelques miniaturistes 5 . A la suite de Morisset, John Russell Harper fait paraître en 1966 son ouvrage La Peinture au Canada, des origines à nos jours, dans lequel il consacre un chapitre à l'art de la miniature et de la silhouette. Puis, en 1970, Harper publie Early Painters and Engravers in
Canada ${ }^{7}$, un dictionnaire des artistes nés avant 1867 et ayant travaillé au Canada, dans lequel sont recensés la plupart des miniaturistes qui avaient annoncé leurs services dans les journaux du pays. Depuis le dépôt du mémoire de Rosenfeld, plusieurs historiens d'art ont étudié la carrière de ces artistes ou ont fait des recherches sur certaines miniatures. Nous ne mentionnerons ici à titre d'exemples que quelques ouvrages majeurs tels que les catalogues d'exposition La Peinture au Québec, 1820 $1850^{8}$, Berczy et Un moment dans l'histoirel ${ }^{10}$, de même que le Dictionnaire des artistes de langue française en Amérique du Nord" ${ }^{1}$. Basé entre autres sur le travail de ces historiens d'art, ainsi que sur les résultats de nos propres recherches entourant notre mémoire intitulé L'Art de la miniature au Bas-Canada entre 1825 et 1850 : Gerome Fassio et ses contemporains ${ }^{12}$, le présent article se veut d'abord une réflexion sur l'objet d'étude que constitue la miniature canadienne, mais aussi une mise en lumière des difficultés inhérentes à la recherche dans ce domaine. Dans le but de restreindre le corpus des ouvres qui serviront ici d'exemples, mais également dans celui de redécouvrir la collection qui constituera le fonds du nouveau Musée du portrait, nous avons choisi de nous concentrer sur les miniatures canadiennes conservées par Bibliothèque et Archives Canada (BAC). Après une brève présentation de cette collection, nous réfléchirons sur les conditions de production des miniatures aux XVIII et XIX ${ }^{e}$ siècles au Canada, puis sur les divers aspects de la relation entre la miniature et son propriétaire initial.

Depuis le début des années 1930, les Archives nationales 
du Canada - maintenant connues sous le nom de Bibliothèque et Archives Canada - ont acquis près d'une centaine de portraits miniatures exécutés au Canada ou ailleurs, et représentant pour la plupart des personnes ayant vécu au pays durant une plus ou moins longue période ${ }^{13}$. Si la majorité de ces miniatures ont été vendues ou cédées à $\mathrm{BAC}$ par des descendants des modèles, plusieurs proviennent de collectionneurs privés ou de galerics d'art. Enfin, une part plus modeste de la collection a été acquise par l'entremise de maisons de ventes aux enchères. L'historique de la collection de BAC est marqué par des dons ou par des ventes de miniatures regroupées en lots. En 1959, Sir Donald Alexander Smith, baron de Strathcona, cède à l'institution nationale une large collection d'objets divers, dont neuf miniatures en émail ou à l'aquarelle sur ivoire. Ces miniatures représentent entre autres des membres de la famille royale britannique. En 1972, les Archives nationales deviennent acquéreurs des quatre portraits miniatures représentant des membres de la famille McGillivray, offerts par la succession d'une descendante par alliance, madame T. A. Dawkins. Sept ans plus tard, l'institution reçoit le don le plus important en nombre - soit quatorze miniatures réalisées à l'aquarelle sur ivoire - de la part des descendants de la famille Hale-Hensley-Amherst. Durant la décennie 1980, la collection se voit augmentée par l'acquisition de trois miniatures provenant des descendants de la famille Gugy-Porteous, ainsi que de trois autres portraits représentant des membres de la famille Ellice (par l'entremise d'une maison de ventes aux enchères). Enfin, en 1989, des descendants de la famille Claus cèdent aux Archives huit portraits miniatures représentant leurs aïeux.

Au sein de la collection, l'identité des personnes portraiturées nous est connue grâce à des inscriptions à l'endos des œuvres ou grâce à la mémoire des descendants. Toutefois, près du quart des portraits miniatures conservés par BAC représentent des modèles non identifiés. Il en va de même quant à l'attribution des œuvres à des miniaturistes; en effet, seules une quarantaine de miniatures ont pu être ainsi attribuées. Ces lacunes rendent à leur tour impossible l'identification du lieu de production des œuvres. Néanmoins, nous estimons qu'environ la moitié des miniatures de la collection de BAC ont été exécutées en Europe, alors que les autres œuvres proviennent du Canada (environ un quart) et des États-Unis (environ un dixième). En ce qui a trait à la datation des ceuvres, lorsque les informations sur le miniaturiste ou le portraituré sont jugées trop parcellaires, certains détails iconographiques, comme ceux relatifs au costume, peuvent renseigner l'observateur attentif. Ainsi, peu de miniatures de la collection ont été exécutées antérieurement à 1760 . En revanche, une trentaine d'entre elles datent de la période s'étendant de 1760 à 1800 . Nous en avons dénombré presque autant pour la première moitié du XIX ${ }^{e}$ siècle, la seconde moitié du siècle s'avérant beaucoup moins fructueuse. Les œuvres réalisées après 1900 constituent près du cinquième $\mathrm{dc}$ la collection.

Comme nous l'avons déjà précisé, Bibliothèque et Archives Canada conserve environ vingt-cinq miniatures susceptibles d'avoir été exécutées au Canada. D'après les renseignements disponibles sur les modèles et les artistes, la plupart de ces miniatures proviendraient du Bas-Canada (Québec), alors que quatre d'entre elles auraient été réalisées dans le Haut-Canada $(\text { Ontario) })^{14}$, deux dans les Maritimes ${ }^{15}$ et une seule en Colombie-Britannique ${ }^{16}$. Les plus anciennes miniatures exécutées au Canada sont datées de la seconde moitié du XVIII e siècle. Notons que près de la moitié des miniatures canadiennes de la collection ont été exécutées au XXe siècle. Ce fait peut paraître étonnant compte tenu que la pratique de la miniature, au Canada comme ailleurs, tend à se raréfier après 1900. Cette surreprésentation des miniatures datant du XXe sic̀cle s'explique par la présence de cinq portraits donnés par la miniaturiste québécoise Juliette de Lavoye (1903-?) ${ }^{17}$ et de deux portraits offerts par la fille de l'artiste britannique Louie Burrell (1873-1971) ${ }^{18}$.

Les conditions de production et la provenance des miniatures : des obstacles à la recherche

Cette brève description de la collection de miniatures de Bibliothèque et Archives Canada rend compre des difficultés que causent les renseignements lacunaires sur l'identité des artistes et des modèles, ainsi que sur les lieux et les dates de production. Parmi les facteurs permettant d'expliquer ces nombreux points obscurs, les conditions de production particulières à cette pratique artistique tiennent un rôle de premier rang. Contrairement aux portraitistes de grand format qui parviennent à mener une carrière relativement stable, les miniaturistes doivent composer avec une situation économique assez précaire. En effet, durant les XVIIIc et XIXe siècles, le nombre toujours croissant de miniaturistes travaillant au Canada et aux États-Unis oblige ces artistes à se déplacer d'un centre urbain à l'autre dans le but de diversifier et de renouveler leur clientèle ${ }^{19}$. Par exemple, de 1760 à $1860^{20}$, une cinquantaine d'artistes professionnels se spécialisant dans la confection de portraits miniatures et de silhouettes, en provenance d'Europe ou des États-Unis, séjournent à Montréal pour des périodes plus ou moins longues ${ }^{21}$.

Nos recherches entourant la rédaction de notre mémoire nous ont permis de distinguer deux catégories de miniaturistes professionnels œuvrant au Canada. S'inscrivant dans la première catégorie, un nombre important de miniaturistes professionnels ne visitent la colonie que pour un laps de temps très court. Ces artistes voyagent d'un endroit à l'autre, au gré des commandes et mènent donc une existence itinérante. Installant leur atelier dans une chambre d'hôtel, ils font paraître une 
Figure I. Charlotte Deming, John Fletcher, 1845, aquarelle sur ivoire, 10,7 ×8,7 cm, Ottawa, Bibliothèque et Archives Canada (crédit photographique : Bibliothèque et Archives Canada, $(-083510)$.

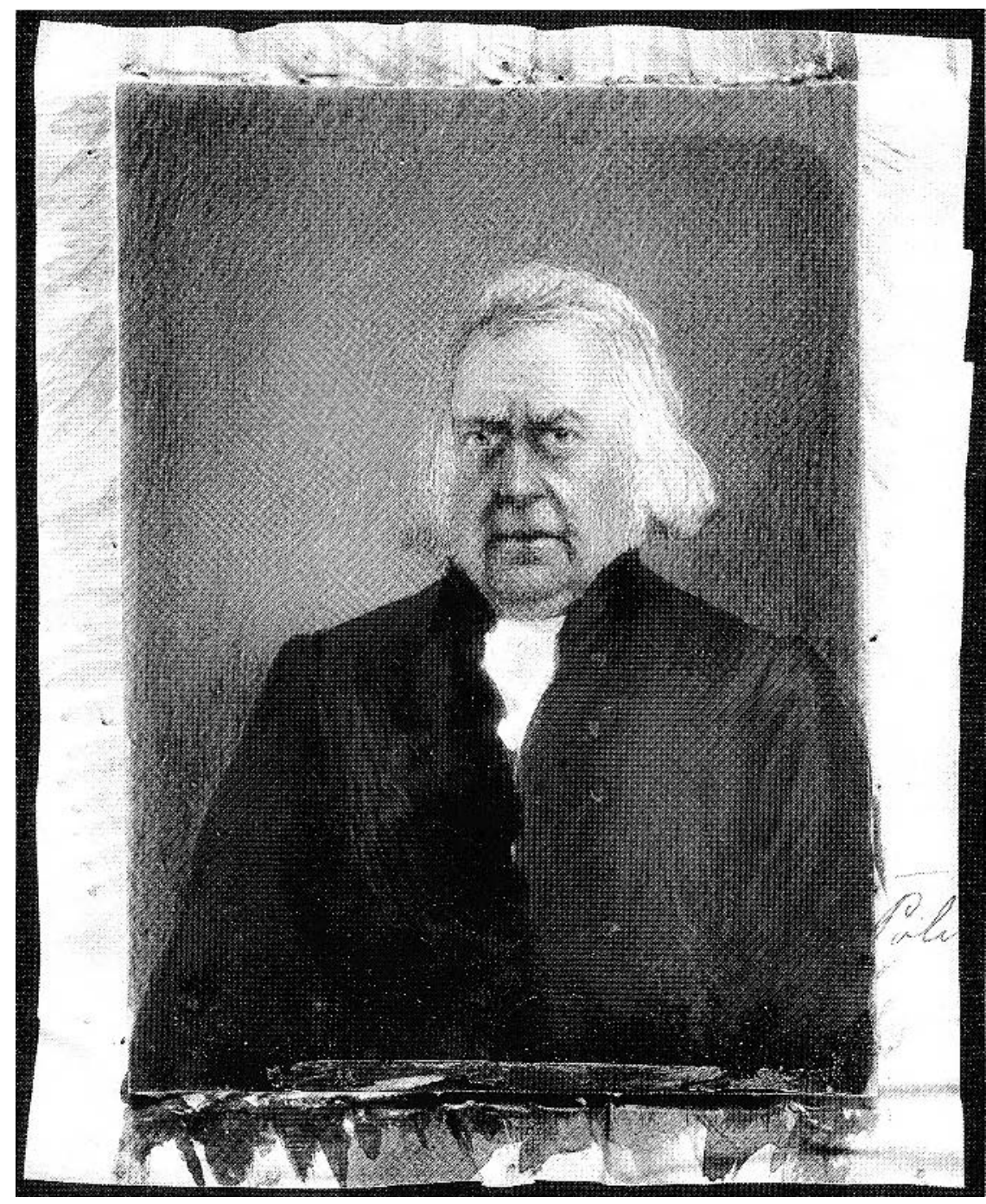

durant une courte période. Le portrait du juge John Fletcher (1767-1844) (fig. 1) comporte, sur le papier protecteur fixé à l'endos de l'œuvre, la signature de Charlotte Deming, l'une des premières femmes miniaturistes professionnelles au Canada ${ }^{24}$. Datant du mois de juillet 1845 , cette effigie posthume a pu être exécutée à partir d'un portrait antérieur ou d'un daguerréotype. Appartenant à la même institution, le portrait présumé de George Matthews (c. 1816-1866) constitue l'une des rares miniatures que l'on a pu attribuer à un artiste grâce à une analyse stylisti$q^{25} e^{25}$. En effet, ce portrait aurait été exécuté par Henry Colton Shumway, un miniaturiste célèbre dont plusieurs œuvres sont conservées dans des musées américains ${ }^{26}$. Graveur, lithographe et copropriétaire de l'entreprise montréalaise Matthews Lithography, George Matthews aurait pu se faire portraiturer à Montréal, lors de la visite de Shumway en 1844 , tout comme il aurait pu commander sa miniature lors d'un voyage d'affaires à New York. La composition plutôt traditionnelle du portrait de Matthews, un buste inséré dans un médaillon ovale, de même que le cadrage très serré de la tête rappellent certaines miniatures réalisées par l'artiste durant les années $1830^{27}$. En outre, l'utilisation de couleurs sobres, le modelé très doux des tons - en particulier dans le rendu de la chair - et la juxtaposition de touches délicates à l'arrière-plan sont autant de traits stylistiques qui justifient l'attribution de l'œuvre à Shumway.

annonce dans les journaux locaux et offrent à l'occasion des cours de dessin ${ }^{22}$. Le prix des œuvres, de même que leur qualité, varient grandement, allant de cinquante cents pour un simple portrait de profil à trois cents dollars pour une miniature sur ivoire de l'éminent artiste américain Henry Colton Shumway (1807-1884 $)^{23}$. L'attribution de miniatures non signées à ces artistes de passage s'avère une opération délicate. D'une part, lors de leur séjour dans la colonie, ils n'ont pu exécuter qu'un nombre assez restreint d'œuvres, dont trc̀s peu nous sont parvenues. D'autre part, ces miniaturistes itinérants sont rarement d'une grande notoriété; leur carrière de miniaturistes est souvent de courte durée et l'absence d'œuvres pouvant leur être attribuées avec certitude empêche toute comparaison significative.

Parmi les œuvres de la collection de BAC, deux miniatures ont été exécutées par des artistes ayant visité le Bas-Canada
En concurrence avec les artistes de passage, les miniaturistes de la seconde catégorie, provenant également de l'étranger, établissent leur résidence permanente dans la colonie et y demeurent durant plus d'une année. Contrairement à leurs compériteurs itinérants, la plupart de ces artistes diversifient leurs activités afin de s'assurer une plus grande part du marché. Leurs offres de services dans les journaux nous apprennent que ces miniaturistes se proposent d'enseigner égalemenmt le dessin ainsi que de réaliser des portraits de grand format, des paysages, des tableaux d'histoire et des lithographies ${ }^{28}$. Quoique les collections canadiennes conservent un nombre important d'œuvres de tous genres exécutées par ces artistes, l'attribution de leurs miniatures n'en est pas facilitée pour autant ${ }^{29}$.

Parmi les miniatures canadiennes de la collection de BAC, une seule œuvre a jusqu’à maintenant été attribuée à un minia- 
Figure 2. William Lockwood (c.1803-1866), attr. à, Portrait d'une inconnue, vers 18601865, aquarelle sur papier, $7 \times 5 \mathrm{~cm}, 0$ ttawa, Bibliothéque et Archives Canada (credit photographique : Bibliothèque et Archives Canada, (-095753).

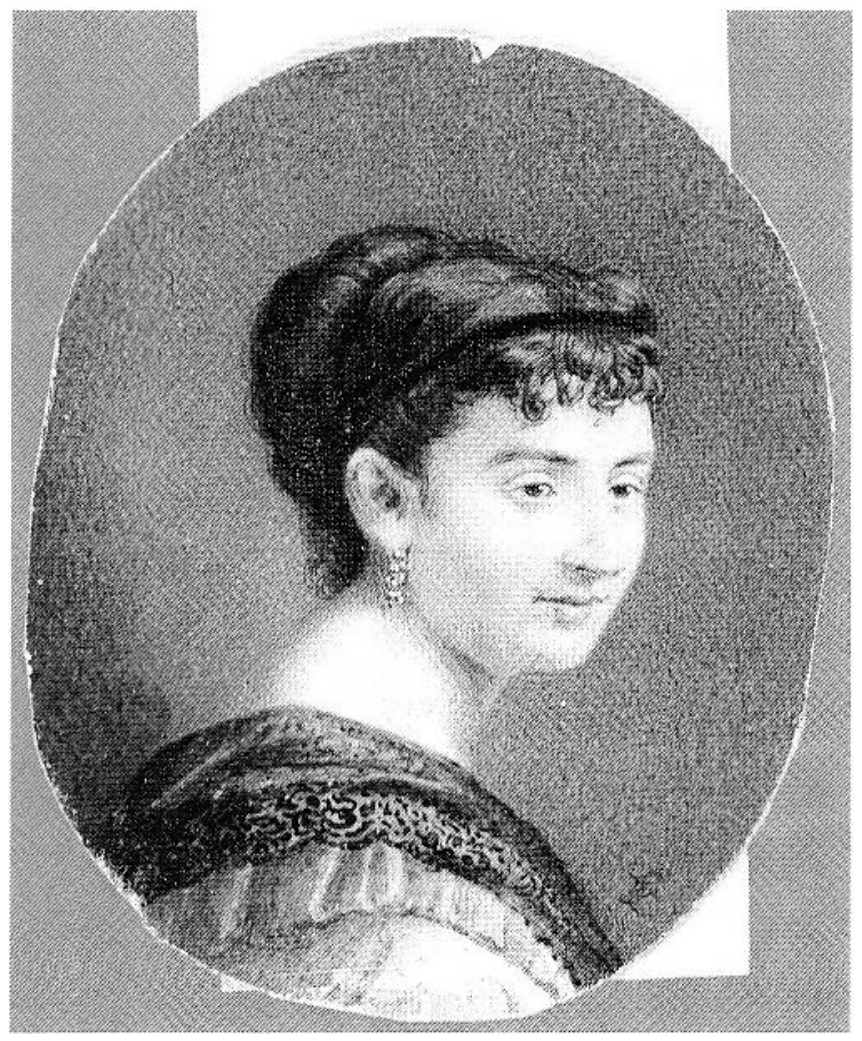

turiste de la seconde catégorie. Le Portrait d'une inconnue (19591-8) (fig. 2), représentant le buste d'une jeune femme, comporte dans l'image le monogramme " $W L$ " qui a permis d'associer l'œuvre à William Lockwood ${ }^{30}$. La miniature de forme ovale a été insérée dans un cadre à l'intérieur duquel apparaissent les insignes ainsi que les coordonnées d'Adolphe Eugène Disdéri (1819-1890), inventeur parisien de la "carte de visite" en 1854 et photographe officiel à la cour de Napoléon III jusqu'en 1866. L'encadrement contient également une carte du magasin montréalais The Art Emporium, alors situé au 357 de la rue Sainte-Catherine Ouest ${ }^{31}$. La datation assez tardive de cette miniature (1860) est basée sur la coiffure du modèle ainsi que sur la pose d'inspiration romantique que l'on peut rapprocher de certains portraits photographiques du temps.

Enfin, nos recherches concernant les miniatures bas-canadiennes nous ont également permis de discerner une dernière catégorie d'artistes. Cette catégorie regroupe des miniaturistes amateurs $^{32}$, c'est-à-dire des hommes et des femmes qui réalisent des miniatures dans un but non commercial. Constituant un groupe hétéroclite et difficile à quantifier, ces amateurs ont eu l'occasion d'apprendre leur art grâce aux cours privés ou semiprivés offerts par les miniaturistes de passage ou dûment établis dans leur ville. Les jeunes filles de l'époque peuvent également apprendre les rechniques de la miniature dans les couvents. Par exemple, en 1842, l'artiste Victor Ernette enseigne cet art aux novices de l'Hôpital général de Québec et initie les Ursulines de Québec ${ }^{33}$ à une grande variété de techniques. Quatre ans plus tard, ces religieuses forment à lcur tour leurs élèves dans plusieurs domaines dont la peinture à l'huile, l'aquarelle et la miniature ${ }^{34}$. La présence au Séminaire de Québec, à titre de professeurs de dessin, des miniaturistes Charles Woodley, entre 1835 et $1839^{35}$, et Gerome Fassio, en 1839-1840 36 , pourrait avoir permis à ces artistes de recruter des élèves pour leurs cours privés.

L'avocat, cartographe et homme politique Robert-ShoreMilnes Bouchette (1805-1879), fils de l'arpenteur général du Bas-Canada Joseph Bouchette (1774-1841), peignait et dessinait durant ses temps libres. Mieux connu pour ses paysages aquarellés ${ }^{37}$, cet artiste amateur a aussi exécuté des autoportraits, de même que la miniature sur ivoire représentant Wolfred Nelson (1791-1863) (fig. 3) conservée à BAC. Des renseignements inscrits sur le papier protecteur à l'endos de la miniature permettent d'identifier la date et le lieu de production, qui correspondent à l'exil du modèle et de l'artiste aux Bermudes suite aux révoltes patriotiques de $1837-1838^{38}$. Au sein de la collection de BAC, un second portrait a été peint par un artiste amateur. Comme l'indique l'inscription à l'endos de l'œuvre, Lady Henriette Martha Hamilton, l'épouse du gouverneur de Terre-Neuve Sir Charles Hamilton, a exécuté la miniature de Demasduwit (Mary March) (c. 1796-1820), une jeune femme de la tribu des Béothuks enlevée par des colons terre-neuviens en 1819 , lors d'une tentative d'apaisement des conflits existant entre les dcux peuples ${ }^{39}$. Alors que la facture de certains portraits peut attester de leur caractère amateur, d'autres, comme le portrait de Demasduwit (Mary March), arrivent à confondre les spécialistes par la qualité du dessin et des techniques utilisées.

En plus des difficultés provenant des conditions particulières dans lesquelles s'est exercée la pratique de la miniature au Canada, le travail des chercheurs se voit entravé par la perte d'informations relatives à la provenance et à la transmission des œuvres. Comme dans le cas des portraits de grand format, les miniatures font partie du patrimoine familial et sont ainsi léguées de génération en génération. Avec le temps, les renseignements concernant le portraituré, l'artiste, la datation, le lieu et les circonstances de la production s'appauvrissent. Les inconvénients reliés à cette perte progressive d'informations peuvent être illustrés par l'étude du portrait de la Femme de la famille Field de Cobourg, Ontario (1990-200-1) (fig. 4), appartenant à BAC. Acquise par l'entremise de Sotheby's Canada, la miniature aurait appartenu, selon la note d'accompagnement signée par John L. Russell, à une descendante de la famille Field établie à Cobourg entre 1830 et 1850 . L'hypothèse selon laquelle cette miniature aurait pu être peinte dans le Haut-Canada est soutenue par la facture très rudimentaire du portrait et par le style du 
Figure 3. Robert-Shore-Milnes Bouchette (1805-1879), attr. à.,Wolfred Nelson, 1838, gouache et aquarelle sur ivoire, $7,4 \times 6 \mathrm{~cm}$, Ottawa, Bibliothèque et Archives Canada (crédit photographique: Bibliothéque et Archives Canada, C-083488)

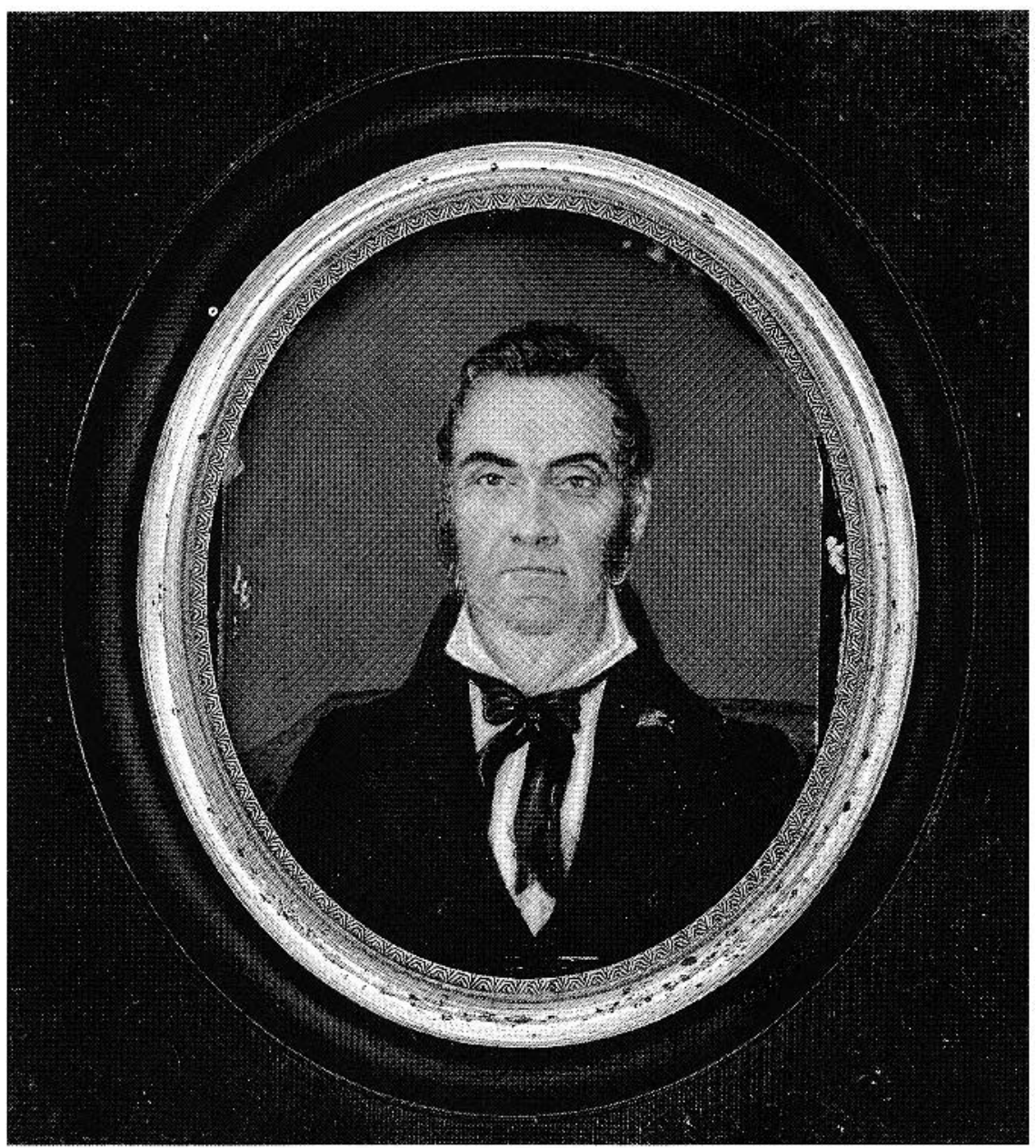

nels qui travaillent au Canada à partir du $\mathrm{XVIII}^{e}$ siècle sont, pour la grande majorité sinon la totalité, des artistes étrangers ayant reçu leur formation en Europe ou aux États-Unis. Si l'analyse de la collection de miniatures canadiennes de Bibliothèque et Archives Canada ne nous permet pas de discerner toutes les caractéristiques propres à chacune de ces étapes, elle peut en revanche nous en révéler quelques aspects.

Avant 1800, au Canada comme ailleurs, la plupart des miniaturistes exécutent des portraits en buste à l'aquarelle sur un support d'ivoire aux dimensions très restreintes. De forme ovale, les miniatures de l'époque sont souvent conçues pour orner des pendentifs, des broches et des bracelets, ou sont enchâssées dans des encadrements richement ouvragés $^{42}$. Parmi les miniatures canadiennes de ce type conservées à $\mathrm{BAC}$, le portrait en buste, présenté de trois quarts, de Sir John Johnson (fig. 5) constitue l'œuvre la plus représentative de la pratique de la miniature à la fin du XVIII ${ }^{e}$ siècle au Canada. Le miniaturiste non identifié a utilisé la technique du pointillé dans le rendu du visage, alors que l'arrière-plan et les vêtements sont exécutés par fines hachures.

Les premières décennies du XIX $\mathrm{X}^{e}$ siècle voient s'amorcer des transformations majeures dans la pratique des miniaturistes. D'abord, la miniature en bijou tend à dispa-

cadre. En contrepartie, les dimensions de la miniature, qui ne correspondent pas exactement à celles du cadre, suggèrent le remplacement de l'encadrement original. De surcroît, la datation du portrait, basée sur l'arrivée de la famille Field à Cobourg, ne coïncide pas à la période suggérée par les vêtements du modèle - en particulier la robe à taille haute - qui évoquent plutôt une période quelque peu antérieure.

\section{Des étapes dans la pratique de la miniature au Canada}

Les études concernant lcs miniatures conservées dans les collections canadiennes, en particulier le mémoire de Roslyn Rosenfeld intitulé Miniatures and Silhouettes in Montreal, 1760-186040, ont permis de discerner certaines étapes dans la pratique des miniaturistes. En fait, le développement de l'art de la miniature au Canada semble suivre sensiblement celui que décrivent les spécialistes de la miniature européenne et américaine ${ }^{41}$. Cette similitude s'explique par le fait que les miniaturistes profession- raître, ce qui permet aux artistes de s'attaquer à des dimensions un peu plus importantes. Les miniaturistes favorisent alors l'emploi de cadres à l'ornementation plus sobre, conçus de façon à permettre l'accrochage du portrait au mur ou son exposition sur une commode. Quoique l'ivoire demeure encore le support le plus prisé, le papier gagne en popularité chez les miniaturistes qui désirent produire des œuvres à moindre coût. À partir de 1825 environ, le traditionnel format ovale se voit peu à peu délaissé au profit d'un support rectangulaire ${ }^{43}$. Enfin, les miniaturistes qui, auparavant, appliquaient de très fines couches d'aquarelle afin de préserver l'aspect translucide de l'ivoire, préferent désormais les couleurs opaques et recherchent l'effet lustré que produit la gomme arabique utilisée comme vernis afin d'imiter les portraits peints à l'huile ${ }^{44}$. Par exemple, dans le portrait de Wolfred Nelson (fig. 3), Robert-Shore-Milnes Bouchette a exécuté à la gouache l'habit noir du modèle et le fond brun très opaque, n'urilisant l'aquarelle que pour le visage.

Au cours des années 1840-1850, les miniaturistes devront 
Figure 4. Miniaturiste non identifie, Femme de la famille Field de Cobourg, Ontario, vers 1830-1850, aquarelle sur ivoire, $6,3 \times 5 \mathrm{~cm}$, Ottawa, Bibliothèque et Archives Canada (crédit photographique : Bibliothèque et Archives Canada, C-137885)

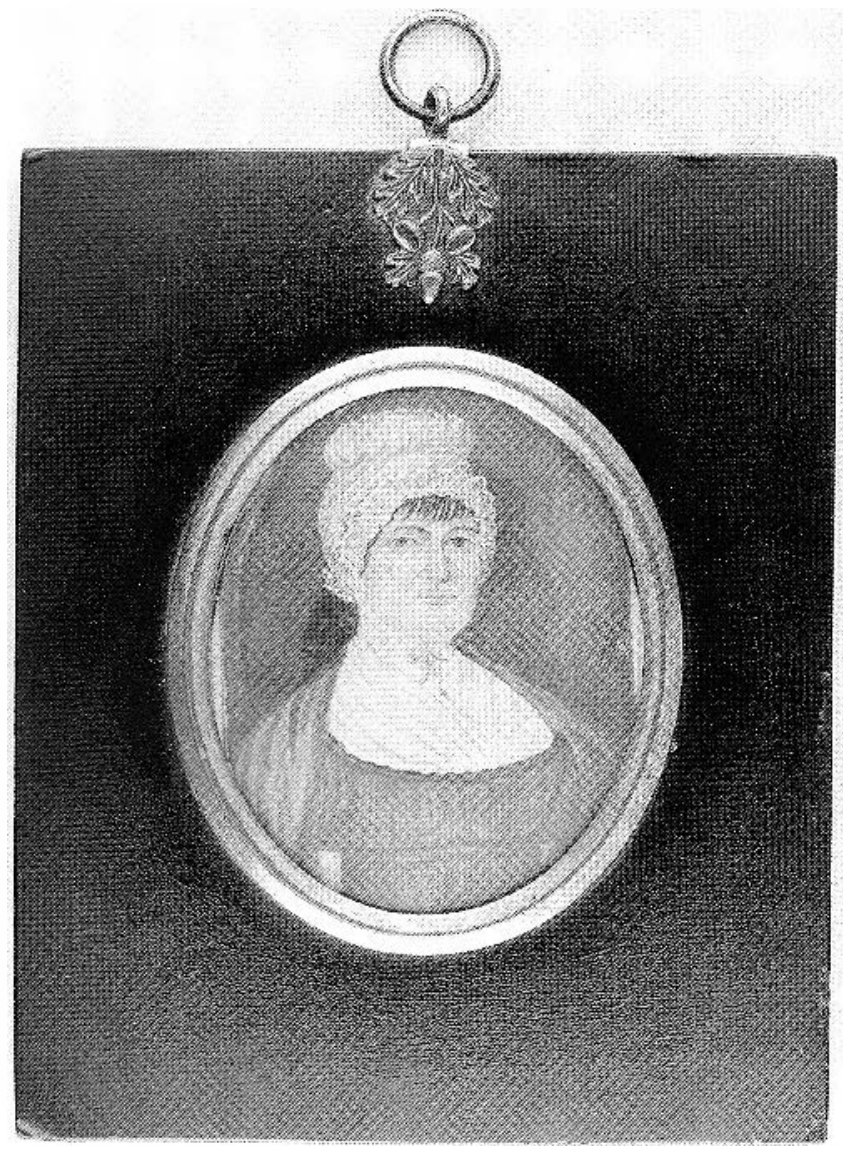

adapter leur pratique aux nouvelles attentes de la clientèle qui peut désormais obtenir un portrait photographique à prix abordable. La compétition toujours grandissante des daguerréotypes, qui supplantera l'art de la miniature durant la seconde moitié du XIX ${ }^{\mathrm{e}}$ siècle, encourage les miniaturistes à poursuivre la transformation de leur pratique amorcée plusieurs années plus tôt sous l'influence des peintres portraitistes ${ }^{45}$. Les daguerréotypes et les ambrotypes présentant sans discrimination les qualités et les défauts des modèles, les miniaturistes vont maintenant chercher à atteindre une plus grande fidélité dans le rendu des physionomies. Finis l'agrandissement des yeux et la réduction du nez que l'on pouvait encore observer dans le portrait de Sir John Johnson (fig. 5); les miniaturistes prennent désormais soin d'éviter ce genre d'écart par rapport à la nature.

L'émulation de la précision photographique semble porter certains peintres et miniaturistes à une finesse accrue du dessin et à une attention plus soutenue aux détails. La minutie du dessin de William Lockwood, de même que l'originalité des poses dont on trouve un bon exemple dans son Portrait d'une inconnue (fig. 4), expliquent sans doute son grand succès au Canada durant les années 1840-1860, et ceci malgré la compé-
Figure 5. Miniaturiste non identifié, Sir John Johnson, vers 1766-1799, aquarelle sur ivoire, $7,7 \times 6 \mathrm{~cm}$, Ottawa, Bibliothéque et Archives Canada (crédit photographique : Bibliothèque et Archives Canada, (-088436).

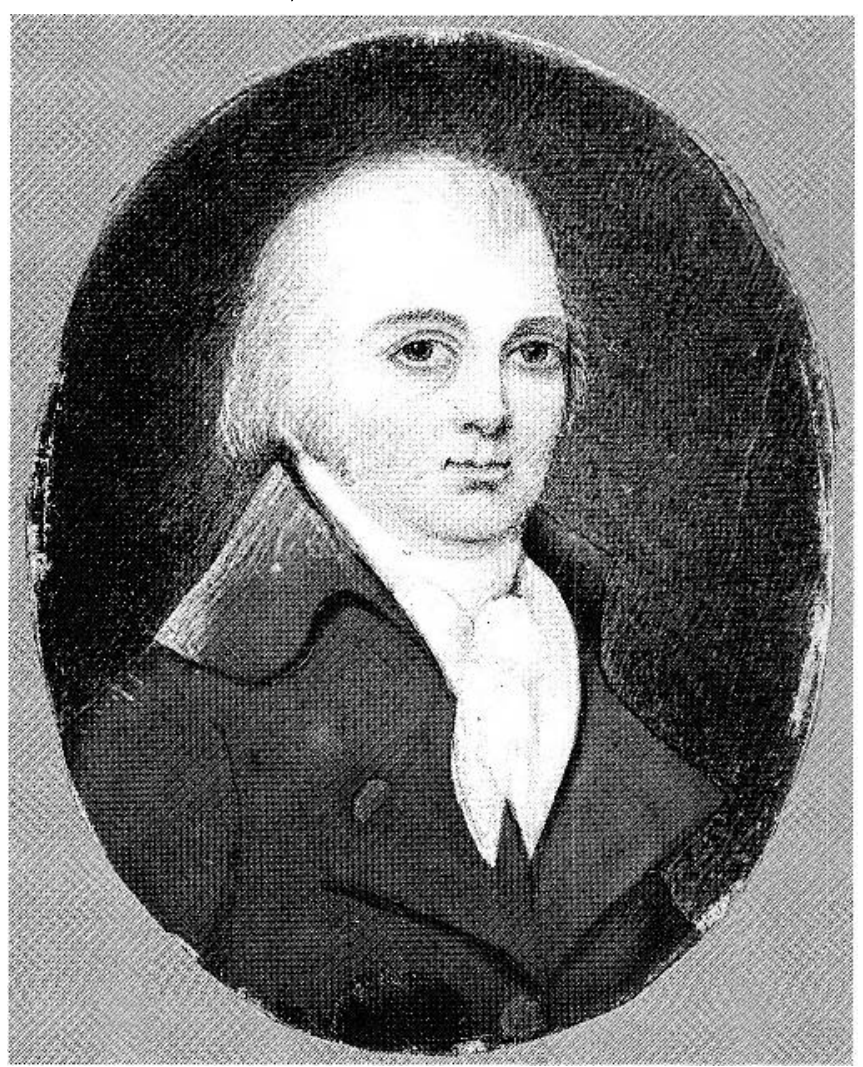

tition des photographes. Enfin, une dernière influence de la photographie se manifeste dans la nouvelle frontalité imposée aux modèles ${ }^{46}$. Cette caractéristique se retrouve assez tôt dans les portraits miniatures comme celui de John Fletcher (fig. 1). Le visage du modc̀le paraît ici crispé et les yeux sont froncés comme dans les premiers daguerréotypes, lorsque la séance de pose durait plusieurs minutes. À l'absence d'idéalisation des traits du modèle s'ajoutent le nouveau format rectangulaire, le cadrage important, les plus grandes dimensions du support pour témoigner de l'influence de la photographie.

De la fin du XIX ${ }^{e}$ siècle au début de la Première Guerre mondiale, la pratique de la miniature connaît un regain de popularité. La collection de miniatures canadiennes de BAC comporte quelques œuvres qui attestent de cette renaissance. Préconisant un retour aux caractéristiques propres à ce genre artistique, les miniaturistes adoptent principalement des formats circulaires et ovales et enchâssent leurs ouvres dans des médaillons pouvant servir de broches ou de pendentifs. Toutefois, la précision presque photographique du dessin demeure un critère d'appréciation majeur pour la clientèle. Du reste, les miniatures de cette époque sont plus souvent exécutées à partir d'un portrait photographique que d'après nature. Par exemple, la miniature rcprésentant Alice Walker (1881-1890) (fig. 6) a 
Figure 6. Horatio Walker, Alice Walker, vers 1891, aquarelle et gouache sur ivoire, $4,5 \times 4,0 \mathrm{~cm}$, Ottawa, Bibliothèque et Archives Canada (crédit photographique : Bibliothèque et Archives Canada, $(-103565)$.

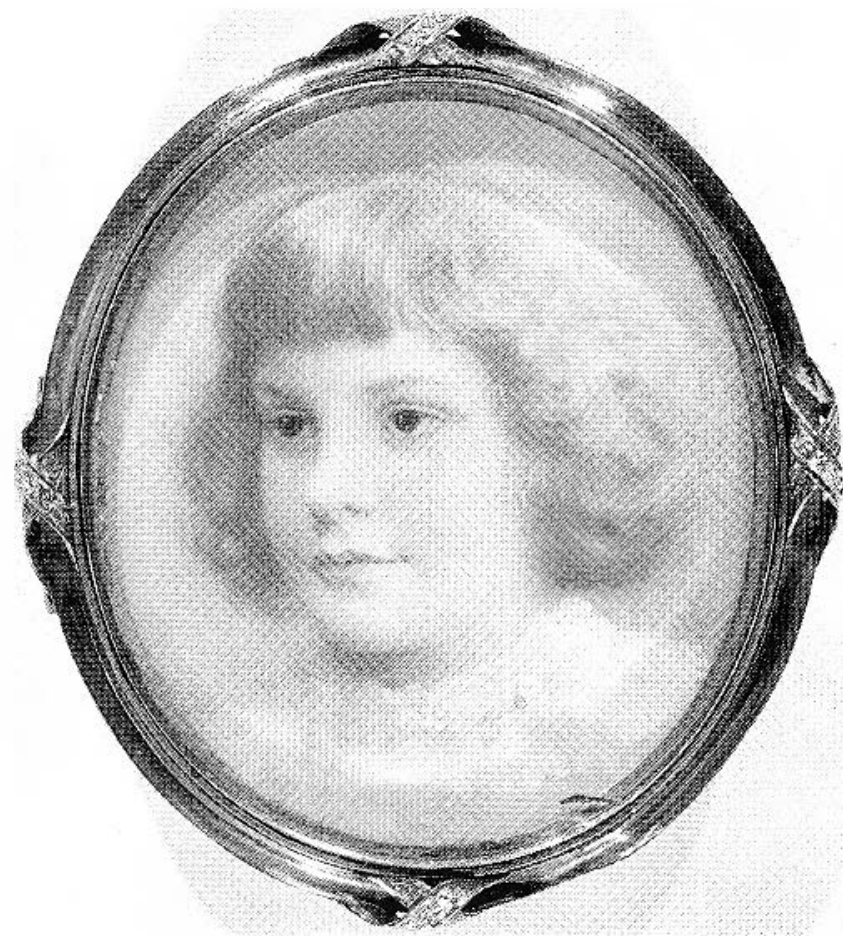

été peinte par son père, Horatio Walker (1858-1938), à la suite du décès de celle-ci, et a donc pu être réalisée d'après une photographie. Ce portrait aux dimensions très restreintes $(4,5 \times 4,0 \mathrm{~cm})$ et enchâssć dans une broche démontre bien le retour à la pratique de la miniature du siècle précédent. La précision photographique recherchée par la clientèle du tournant du siècle se retrouve ćgalement dans le portrait de l'homme politique Cecil Rhodes (1853-1902), exécuté par un artiste non identifié après la mort du modèle. Enfin, la miniature de Sir Wilfrid Laurier (1841-1919), de la main de l'artiste ontarien Gerald Sinclair Hayward (1845-1926), constitue sans doute une copie du portrait photographique réalisé vers 1890 par le Studio Topley, ou de l'une des estampes produites par photogravure d'après ce portrait ${ }^{17}$. Il paraît intéressant de remarquer que le seul portrait au rendu plus inventif, en termes de coloris et de composition (il comporte un arrière-plan historié), correspond à la miniature de Marie Madeleine de Verchères, un portrait historique imaginé par Hayward et devant servir d'illustration à l'ouvrage A Daughter of New France d'Arthur Doughty (1860-1936) ${ }^{48}$.

Les diverses facettes du lien unissant le propriétaire initial à sa miniature

L'un des intérêts majeurs de l'étude de l'art de la miniature, au Canada comme ailleurs, réside dans l'analyse des divers rôles joués par ces petits portraits dans la vie intime et sociale des premiers propriétaires. Des sources textuelles et iconographiques de l'époque, ainsi que les caractéristiques propres à certains encadrements, permettent de comprendre la miniature comme objet de mémoire, comme objet fétiche et parfois, également, comme élément de parure. La collection de miniatures canadiennes de Bibliothèque et Archives Canada fournit des exemples des divers types de liens unissant le propriétaire initial à cet objet particulier qu'est la miniature.

\section{L'objet de mémoire}

Avant l'invention de la photographie, les portraits dessinés, gravés, peints, moulés ou sculptés offraient autant de procédés pour sauvegarder les traits d'un individu ayant vécu à une époque donnće. Le genre du portrait aurait été, depuis ses origines, associé à un désir profondément humain de vaincre la mort et de laisser une trace dans la durée.

Sans doute davantage que le portrait de grand format destiné en partie à la reconnaissance sociale, la miniature relevant du privć appartient au domaine du souvenir. Par ses qualités spécifiques, et principalement par ses dimensions restreintes, la miniaturc établit avec le spectateur un rapport particulier à fortes connotations symboliques. Comme la plupart des objets miniaturisés étudiés par Susan Stewart dans son ouvrage On Longing. Narratives of the Miniature, the Gigantic, the Souvenir, the Collection ${ }^{49}$, le portrait miniature présente un univers hermétiquement clos. Ses limites physiques sont définies de façon évidente par l'encadrement - qui peut prendre diverses formes (du simple cadre en bois au médaillon serti de perles et inséré dans un bracelet) - et souvent par un verre protecteur. Afin de franchir ces limites et d'entrer dans le monde privé et intime de la miniature, l'observateur doit abandonner les soucis de la vie quotidienne et aborder une sorte d'univers intemporel, empreint de nostalgie, qui s'apparente au domaine de la rêverie.

Appelant à la consultation discrète plutôt qu'à la contemplation publique, la miniature se voit attribuer une fonction commémorative; elle évoquc un sentiment, une promesse, un événement ou un accomplissement particulier. Au Canada comme en Europe et aux États-Unis, la réalisation et l'offrande d'une miniature accompagnent les moments importants de la vie du modèle et du propriétaire de l'œuvre ${ }^{50}$. Lors de fiançailles ou d'épousailles, les amants se font portraiturer et s'échangent leur effigic cn signe d'amour et en gage de fidélité. Un portrait miniature intitulé La Mariée (M981.105.59.1), conservé au Musée McCord d'histoire nationale, présente une jeune femme revêtant sa tenue de noces; il comporte, gravée à l'endos du médaillon, l'inscription suivante dont la signification a depuis été oubliée : "Remember Dear Laura". Appartenant à Bibliothèque et Archives Canada, la miniature d'origine européenne représentant Katherine Jane Ellice (c. 1814-1864) a été offerte 
Figure 7. Miniaturiste non identifié, Louis-Christophe-Hilarion Fromenteau, 1800, aquarelle sur papier, 9,1 $\times 7,2 \mathrm{~cm}$, Ottawa, Bibliothéque et Archives Canada (crédit photographique : Bibliotheque et Archives Canada, (-125656).

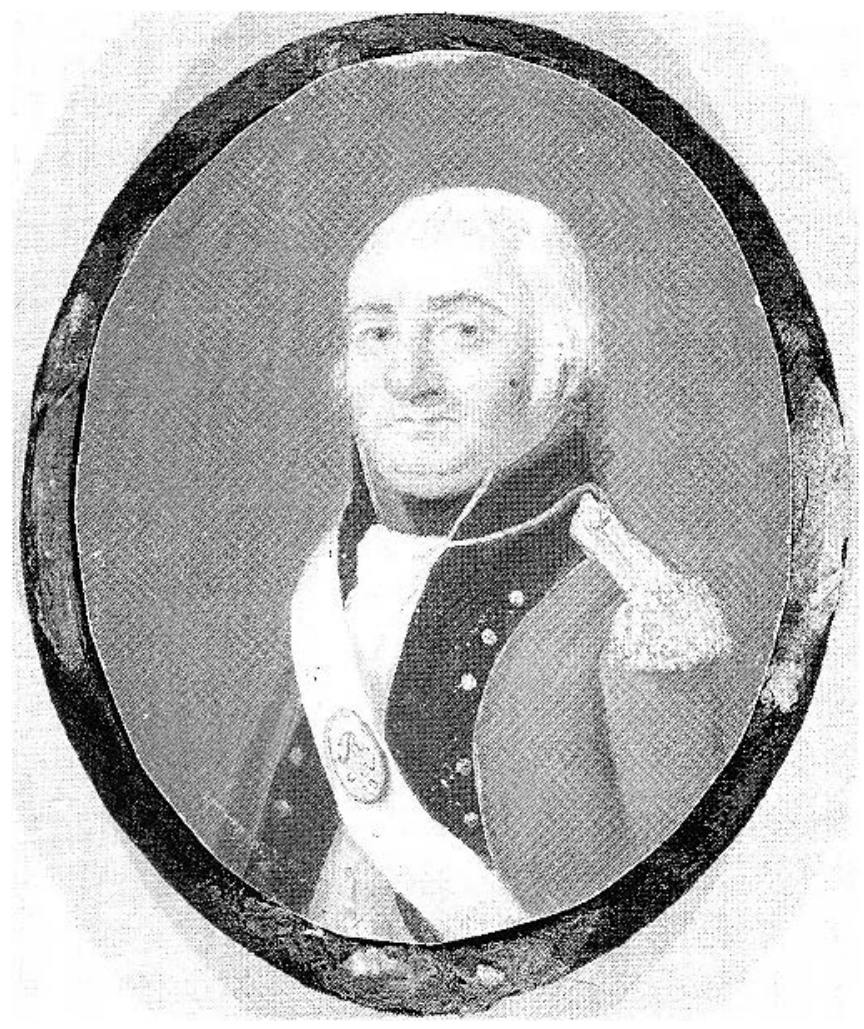

par le modèle à son époux, à l'occasion de leur anniversaire de mariage en juillet 1841 .

De même, les hommes font souvent "tirer " leur portrait, en petit et en grand format, pour souligner une promotion, l'obtention d'une décoration honorifique ou quelque autre événement marquant de leur carrière. Par exemple, il est probable que Louis-Christophe-Hilarion Fromenteau, dont l'effigie se trouve à BAC (fig. 7), se soit fait portraiturer pour célćbrer sa nomination au poste de quartier-maître du $1^{\text {cr }}$ Bataillon des Royal Canadian Voluntecrs. C'est du moins ce qu'on déduit du fait qu'il porte son uniforme militaire. À partir du XVIIIe siècle, les parents jugent de plus en plus important de faire exécuter les portraits miniatures de leurs enfants à cause du taux élevé de mortalité infantile qui sévit à l'époque ${ }^{51}$. BAC conserve plusieurs de ces petits portraits, notamment la miniature intitulée Trois enfants non identifiés (familles Amberst-Hale) (197914-2) (fig. 8, 9), de provenance inconnue et datant de 17661833; la miniature représenterait peut-être les fils de William Pitt Amherst. Le portrait d'un autre héritier Amherst, Enfant non identifié (familles Amherst-Hale) (1979-14-1), de dimensions très restreintes $(4,4 \times 3,7 \mathrm{~cm})$, montre un jeune enfant portant le vêtement à encolure dégagée typique de la fin du XVIII' siècle.

Jusqu'à la fin du XIX ${ }^{e}$ siècle, le taux élevé de mortalité
Figure 8. Miniaturiste non identifie, Trois enfants non identifiés (familles Amherst-Hale), vers 1766-1833, aquarelle et cheveux humains sur tissu, 4,3 $\times 3,6 \mathrm{~cm}$, Ottawa, Bibliotheque et Archives Canada (crédit photographique : Bibliothèque et Archives Canada, C-112073).

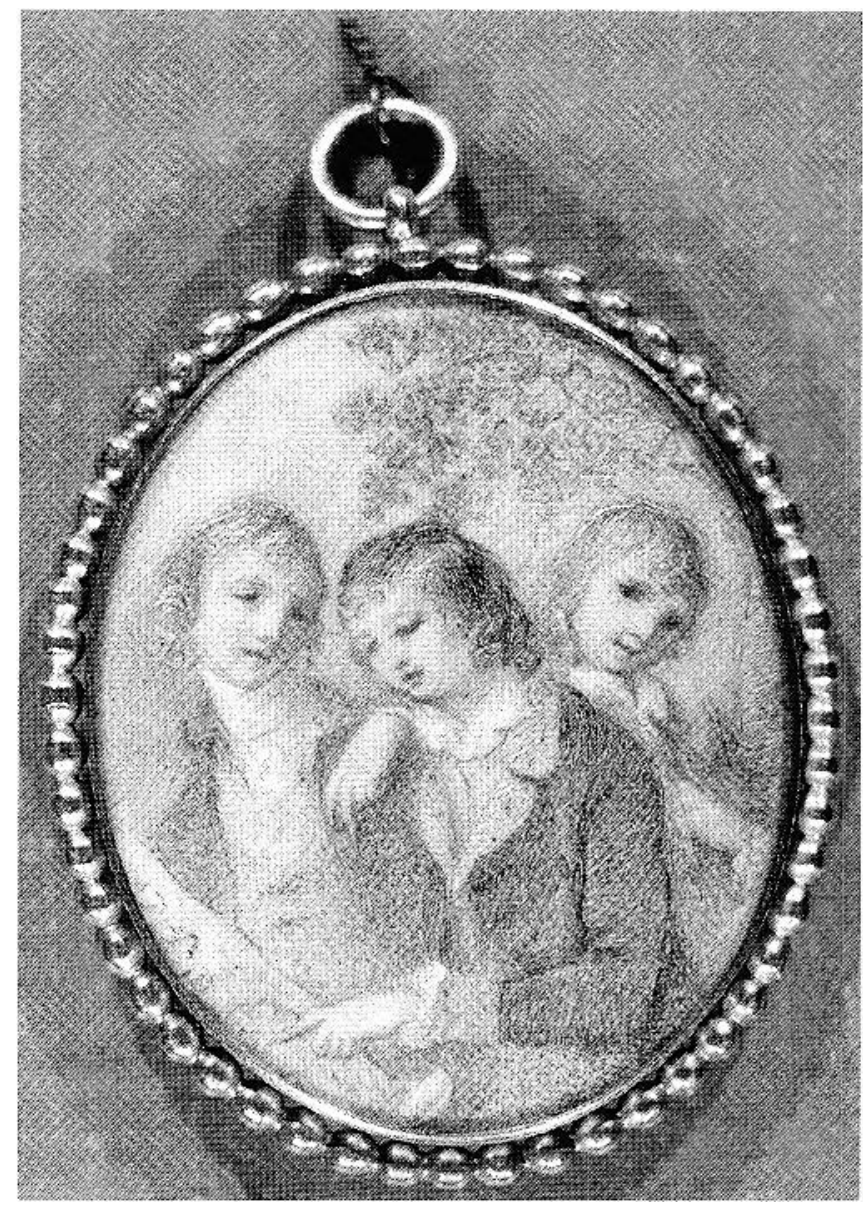

affecte d'ailleurs tous les groupes d'âge de même que toutes les couches de la société. À la perte successive de membres de la famille et d'amis, les gens répondent par l'acquisition ou par la fabrication d'objets de deuil, dont les miniatures. Ces miniatures peuvent être réalisées en direct, c'est-à-dire à partir de la personne défunte, s'inspirer d'un autre portrait ou être exécutées de mémoire ${ }^{52}$. Ce type de portrait posthume affiche souvent des caractéristiques particulières. Plusieurs des miniatures portent simplement, à l'arrière du médaillon, une inscription gravée rappelant les dates de naissance et de décès de l'individu portraituré. D'autres présentent plutôt un motif symbolisant le deuil. Cette pratique, très populaire en Angleterre et aux ÉtatsUnis, a mené à la création de motifs mortuaires standardisés, tels que l'urne, le monument funéraire, le saule pleureur ou la figure féminine éplorée ${ }^{53}$. Toutefois, il ne semble pas que ces images aient connu un grand succès au Canada où l'on n'en trouve que très peu d'exemples ${ }^{54}$. Parmi les miniatures posthumes conservées à BAC, mentionnons le portrait de John Fletcher (fig. 1) exécuté par Charlotte Deming, un an après le décès du modèle. De même, la miniature représentant Alice Walker (fig. 6) 
Figure 9. Miniaturiste non identifié, verso de Irois enfants non identifiés (familles Amherst-Hale), vers 1766-1833, aquarelle et cheveux humains sur tissu, $4,3 \times 3,6 \mathrm{~cm}$, Ottawa, Bibliothèque et Archives Canada (crédit photographique : Bibliothèque et Archives Canada, (-I12074).

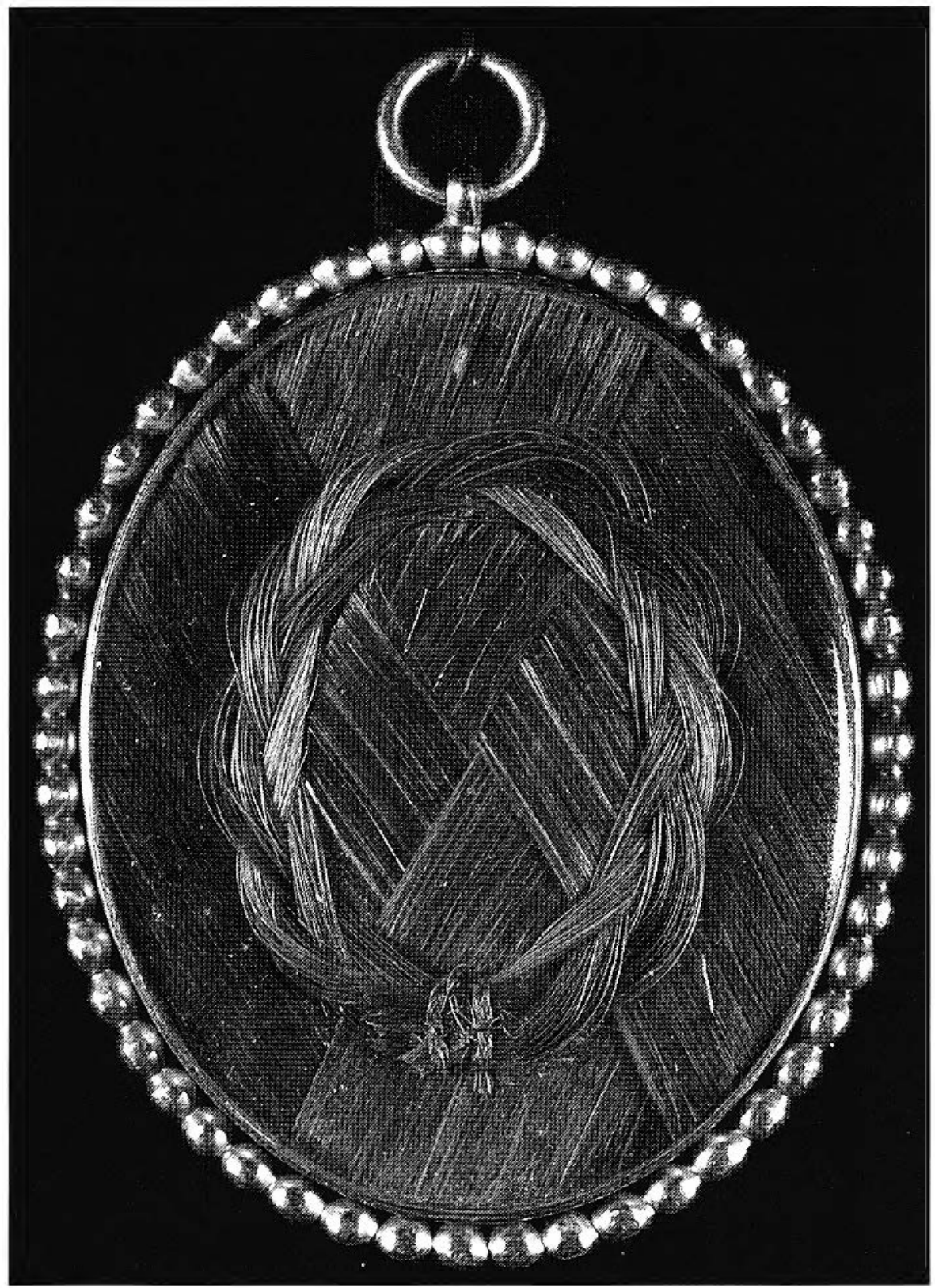

matériaux utilisés. Empruntant d'abord à l'enluminure, les premiers miniaturistes ont recours à un support de vélin ou de parchemin qu'ils appliquent sur des cartes à jouer. Puis le XVII e siècle connaît l'essor de la miniature en émail qui implique l'emploi d'une surface d'or, d'argent ou de cuivre sur laquelle le miniaturiste dépose ses pigments et les fait cuire ${ }^{55}$. Enfin, l'innovation la plus significative pour le développement de la pratique de la miniature est introduite par une artiste du XVIII ${ }^{e}$ siècle, Rosalba Carriera (1675-1757), qui propose de peindre à l'aquarelle sur ivoire ${ }^{56}$. Ces divers supports fournissent aux miniatures leur caractère précieux, qui se voit souvent renforcé par le travail du cadre.

Parce que ces objets fragiles sont produits dans le but d'être aisément transportables, le cadre ou le boîtier dans lequel ils s'insèrent présente un intérêt particulier. À l'opposé des tableaux de grandes dimensions, l'encadrement d'une miniature fait souvent partie intégrante de l'œuvre. Ces considérations ont d'ailleurs mené l'historienne d'art britannique Marcia Pointon à adopter l'expression portrait-object en référence aux miniatures du XVIII' siècle anglais, cette expression devant souligner l'interaction entre l'image et son encadrement ${ }^{57}$. Les premiers miniaturistes produisent des encadrcments en or émaillé souvent ornés de pierres précieuses ${ }^{58}$. Relevant autant de l'art que de la joaillerie, ces miniatures jouent bientôt le rôle de bijou que l'on peut porter en colliers, en pendentifs à la taille ou comme broches.

aurait ćté réalisée par le père du modèle, Horatio Walker, à la suite de la mort prématurée de sa fille atteinte de la diphtéric. Gravée à l'endos du médaillon, une inscription consigne l'identité du modèle ainsi que ses dates de naissance er de décès. L'institution détient également une miniature posthume du politicien Cecil Rhodes.

\section{L'objet de parure}

Dès ses origines au XVI ${ }^{e}$ siècle, l'art de la miniature se distingue du portrait de grand format par la diversité des techniques et des
Alors que les encadrements deviennent un peu plus modestes au XVII' siècle ${ }^{59}$, leur fonction de parure ne s'atténue pas et connaît même son âge d'or au siècle suivant.

Dans le Nouveau Monde, les immigrants français et anglais qui en ont les moyens financiers continuent, malgré l'éloignement, à suivre les modes européennes. Le port de la miniature en bijou connaît donc un certain succès dès la colonisation française, quoique les œuvres et leur cadre proviennent essentiellement d'Europe. Après la Conquête britannique, de plus en plus de miniatures sont créées sur place, alors que les encadrements demeurent pour la plupart des importations. A l'instar de 
nombreux tableaux européens datant des XVII ${ }^{e}, \mathrm{XVIII}^{\mathrm{e}}$ et XIX ${ }^{\mathrm{c}}$ siècles, certains portraits exécutés au Canada révèlent différentes façons de porter les miniatures en bijou. Dans le double portrait de Madame René-Édouard Caron, née Joséphine de Blois, et sa fille Ozine (47.129) conservé au Musée national des beauxarts du Québec, Théophile Hamel (1817-1870) semble avoir reproduit au poignet de la dame un portrait miniature retenu par un bracelet en ruban. Dans la même collection, le portrait de Madame Eustache-Ignace Trottier dit Desrivières, née Marguerite-Alexis Mailhot (56.298), réalisé par François Beaucourt (1740-1794), montre le modèle portant une miniature en pendentif. Dans une notice du catalogue La Peinture au Québec, Eva Major-Marothy précise que la miniature représentée dans le tableau de Beaucourt pourrait être le portrait du fils du modèle, Basilique-Benjamin Trottier Desrivières Beaubien (1988-42-2), l'œuvre de James Peale désormais conservée à $B A C^{60}$.

Au sein de la collection de miniatures de BAC, certaines œuvres sont enchâssées dans des médaillons qui peuvent se transformer en bijoux. Unc majorité de ces médaillons comportent à leur sommet un anneau qui permet de porter l'objet en pendentif. Tel est le cas de la miniature de l'homme d'affaires du Nouveau-Brunswick William Taylor (1789-1834). Quant au Portrait présumé de William Pitt Amherst, datant de la dernière décennie du XVIII e siècle, il est inséré dans un médaillon destiné à être monté sur un bracelet. D'aspect très élaboré, le médaillon contenant le Portrait présumé du Colonel Bartholomew Gugy, serti de cristaux de roche, possède l'épingle et le fermoir lui permettant d'être porté en broche. Au Canada comme ailleurs, le port de la miniature en bijou tend à disparaître à partir du deuxième quart du XIXc siècle, alors que le format de ces portraits s'agrandit. Toutefois, vers la fin du siècle, au moment de la renaissance de l'art de la miniature, la mode refait surface. À cette époque, les portraits photographiques" et les miniatures sont montés en pendentifs, bracelets et broches, comme en témoigne la miniature d'Alice Walker (fig. 6).

Parmi tous les objets d'art décoratif, les bijoux possèdent sans doute la plus grande valeur symbolique. Ils ont historiquement été investis de diverses significations, jouant parfois le rôle de talismans, d'amulettes ou de porte-bonheur. Cette valeur symbolique des bijoux est particulièrement sensible lorsqu'ils font l'objet d'un don à un proche. En effet, le port d'un portrait en bijou témoigne généralement du lien étroit unissant le propriétaire de l'objet au modèle représenté. De façon plus spécifique, la valeur symbolique de la miniature insérée dans un bijou s'apparente à celle de la bague ou de l'anneau. Entre fiancés ou époux, l'échange de portraits miniatures officialise un engagement, rend publique une relation de caractère intime. Cependant, il est à noter que les miniatures enchâssées dans des bijoux font surtout partie, selon la convenance, de la toilette féminine.

\section{L'objet fétiche}

Étymologiquement, le mot fétiche provient du terme portugais feitiço, qui signifie artificiel, lui-même dérivant du latin facticius. Utilisé à l'origine dans le sens d'artificiel ou d'enchanté, le terme fétiche acquiert au XIX ${ }^{\mathrm{e}}$ siècle ses connotations actuelles. À la suite de l'observation des cultes de certaines tribus africaines, les anthropologues nomment fétiches les objets que l'on croit animés d'un esprit et investis d'une force surnaturelle. Marx s'appropriera le terme pour qualifier le pouvoir de la marchandise, un pouvoir basé sur la valeur d'échange plutôt que sur la valeur d'usage de l'objet. Enfin, Freud conférera au fétichisme sa dimension psychanalytique en percevant, dans la relation entre l'individu et son fétiche, la surestimation sexuelle d'un objet non sexuel ${ }^{62}$. Ces diverses acceptions du mot se recoupent dans leur caractérisation du fétiche en tant qu'objet de la vie quotidienne investi par un individu ou une collectivité d'une valeur symbolique inestimable. Dans le catalogue d'exposition intitulé Fétiches \& Fétichismes, Jean-Michel Ribettes propose une réflexion intéressante sur la nature du fétiche: "Le fétiche se caractérise d'abord comme l'incarnation matérielle d'une valeur immatérielle [...], [et] ensuite comme une survalorisation de l'objet immatériel auquel il se substitue ${ }^{63}$. "Ribettes perçoit ainsi dans certains objets anodins, tels un porte-bonheur, une médaille consacrée, une bague de fiançailles ou une photographie d'un proche conservée dans le portefeuille, une réminiscence du fétiche dans la culture occidentale ${ }^{64}$.

Selon la définition de Ribettes, la miniature sc ferait fétiche lorsque, représentant une affection partagée par le modèle et par le propriétaire de l'objet, le portrait devient inconsciemment, aux yeux de ce dernier, la matérialisation de ce sentiment. C'est bien sûr en l'absence de l'être aimé que la miniature se transforme en fétiche. Ribettes insiste d'ailleurs sur ce qu'il nomme $l$ ' " inapaisable métonymie " du fétiche.

Engendré par une pure foncrion de substitution, le fétiche est le représentant d'une valcur absolue, dont il symbolise l'absence, la carence, le déficit [...]. Or, cette valeur qui manque apparaît comme invariablement absolue, souveraine, universelle - l'instance qu'incarne le fétiche est universellement surestimée, surévaluée, surinvestie par le désir des hommes ${ }^{65}$.

Dans la littérature européenne des XVIII et XIXe siècles, la miniature apparaît souvent comme le substitut d'un proche absent ${ }^{66}$. Moins féconde à cet égard, la littérature canadienne a néanmoins laissé quelques témoignages du lien unissant une miniature à son propriétaire. Au mois de mars 1831, un miniaturiste du nom de J. Wilson, installé depuis l'année précédente à Montréal, publie dans la nouvelle revue The 
Montreal Monthly Magazine, dont il est le rédacteur en chef, un poème s'intitulant "To a miniature picture (of an adopted Sister) ". À défaut de pouvoir s'adresser à sa sœur Angelina qui est sans doute demeurée dans leur pays d'origine, l'artiste interpelle son portrait miniature, et ce malgré un faible niveau de ressemblance.

\footnotetext{
Thou faint resemblance, of a sister dear,

To thee I am going to speak, prepare to hear, In vain thy various tints, conspire to vie, With Angelina's lovely sparkling eye, But when I am absent, from that lovely face, With anger eye, I fondly stoop to trace, Each well form'd featurc, gladly I survey, As eye, meets eye, it says, or seems to say, Welcome adopted brother, be at rest ${ }^{67}$.
}

Au XVIII siècle, en Amérique du Nord comme en Europe, se développe un engouement pour les miniatures "reliquaires $^{68}$ ". La plupart de ces miniatures contiennent en effet dans leur étui ou médaillon, à l'endos du portrait, une mèche de cheveux, joliment disposée, appartenant au portraituré ou à l'un de ses proches. Ce type de miniature encourage particulièrement la relation fétichiste. Au sein de la collection de miniatures de Bibliothèque et Archives Canada, quelques œuvres correspondent à ce type d'" ouvrage en cheveux ». Ces miniatures datent pour la plupart de la fin du XVIII ${ }^{e}$ siècle, quoique cette mode ait perduré durant le XIX ${ }^{e}$ siècle. Chacune de ces œuvres a été enchâssée dans un médaillon transformable en pendentif. D'après le rendu de ces miniatures et les informations concernant les modèles représentés, il est fort probable que ces œuvres, de même que leur encadrement, aient été fabriquées à l'étranger. D'ailleurs, de tels ornements connaissent une très grande popularité en France, en Angleterre et aux États-Unis, où des " artistes en cheveux " se spécialisent dans ce genre d'ouvrage ${ }^{69}$.

À l'endos de la miniature représentant Trois enfants non identifiés (familles Amberst-Hale) (1979-14-2) (fig. 8, 9), un " ouvrage en cheveux " sous verre se compose d'une fine tresse circulaire placée sur un fond au motif de mèches superposées. Caractéristique intéressante, des cheveux dissous ont également été utilisés en tant que pigment pour le rendu de la chevelure des trois enfants ${ }^{70}$. De même, à l'arrière du médaillon contenant le portrait d'un Jeune homme non identifié (R231-1), une lunette montre une mèche de cheveux arrangée en motif de palmier et retenue par un fil doré orné de perles. La miniature du Colonel William Claus (1989-407-5) comporte également une lunette révélant le monogramme "CDC", superposé à un motif de cheveux losangé. L'" ouvrage en cheveux " décorant l'arrière du médaillon de la miniature de l'Officier non identifié du 96e Régiment (R5286-1) reprend ce même motif losangé.

Outre la redécouverte des miniatures canadiennes de la collection de Bibliothèque et Archives Canada, le présent article visait une meilleure compréhension de la miniature canadienne en tant qu'objet d'étude, tout en mettant en lumière les difficultés qu'elle peut poser au chercheur. Dans un premier temps, nous avons montré que les conditions dans lesquelles doivent œuvrer les miniaturistes au Canada permettent d'expliquer en partie la perte d'informations importantes concernant les miniatures, telles que le lieu de production, la datation ainsi que l'identité du modèle et de l'artiste. Les miniaturistes professionnels de passage au Canada ne parviennent à réaliser qu'un nombre restreint d'cxuvres, alors que ceux qui s'installent en permanence au pays diversifient leurs services et réduisent donc pour la plupart leurs activités de miniaturistes. Parmi les collections de miniatures conservées au Canada, un nombre difficilement quantifiable d'œuvres ont été produites par des artistes amateurs, souvent des élèves de miniaturistes professionnels. Ces portraits peuvent parfois surprendre par leur qualité. Dans un second temps, nous avons vu que le développement de la pratique de la miniature au Canada suit sensiblement les mêmes étapes qu'en France, en Angleterre et aux États-Unis. Du très petit portrait de forme ovale peint à l'aquarelle sur ivoire et inséré dans un bijou ou un encadrement orné, la miniature se transforme pour ressembler davantage aux portraits de grand format, puis aux portraits photographiques. La renaissance de la miniature vcrs la fin du XIXe siècle voit les œuvres revenir aux caractéristiques plus spécifiques à ce genre, tout en conservant la nouvelle précision presque photographique du dessin. Dans un dernier temps, nous avons étudié les divers rôles pouvant être joués par la miniature dans la vie intime ou sociale du propriétaire initial. Objet de mémoire, la miniature se voit souvent attribuer un rôle de commémoration d'événements importants, heureux ou malheureux. Objet de parure, elle se fait ornement de pendentif, de bracelet ou de broche dans la toilette féminine. Objet fétiche, la miniature est investie par son propriétaire d'une symbolique personnelle qui lui attribue un caractère presque sacré. Au terme de cette étude, ce qu'il importe de retenir concerne le potentiel documentaire d'une recherche poussée prenant comme objet les portraits miniatures.

\section{Remerciements}

Cet article n'aurait pu voir le jour sans la précieuse collaboration de Eva Major-Marothy, conservateur principal des acquisitions et recherches au Musée du portrait du Canada, ainsi que de Martha Marleau, archiviste au département des acquisitions et de la recherche en art à BAC, qui ont facilitć les recherches 
concernant les miniatures de Bibliothèque et Archives Canada. De plus, l'auteure tient à remercier Laurier Lacroix pour ses judicieux conseils.

\section{Notes}

1 Dans cet article, le portrait miniature sera défini comme étant un objet aux dimensions restreintes, c'est-à-dire pouvant être tenu dans la main ct représentant une figure humaine rćalisée à la peinture (huilc, aquarellc, gouache, etc.) à l'aide d'un pinceau et appliquée sur un support quelconque (ivoire, bois, carton, toile, etc.). Le terme miniature, qui réfere parfois à des œuvres de petites dimensions illustrant un paysage ou une naturc morte, par exemple, sera utilisé ici comme synonyme de l'expression " portrait miniature " dans le but d'alléger le textc.

2 Dans son essai intitulé "Regards sur la ressemblance : le portrait au Bas-Canada ", laul Bourassa s'intéresse au coût des différents types de portraits. L'auteur explique que si les miniatures sur ivoire coûtent relativement cher, avec lcs " petits dessins aquarellés ou coloriés au crayon et au pastel sur papier, le portrait devient accessible à une plus grande part de la population ". La silhouette constitue le type de portrait le moins dispendieux. Paul Bourassa, " Regards sur la ressemblance : le portrait au Bas-Canada ", dans $L a$ Peinture au Québec: 1820-1850, Québec, Musée du Québcc, 1991 , p. 44.

3 Roslyn M. Roscnfeld, Miniatures and Silhouettes in Montreal, 1760 1860, Montréal, mémoire, Université Concordia, 1981, 166 p.

4 Gérard Morisset publie, cntre autres, un article intitulé " Guiseppe Fascio le miniaturiste ", La Patrie, 9 avril 1950, p. 26-38.

5 Dans cet ouvrage, Gérard Morisset traite des miniaturistes William Berczy, James D. Duncan, Victor Ernette et Guiseppe (Gerome) Fassio. Gérard Morisset, La Peinture traditionnelle au Canada français, Montréal, Cerclc du livre de France, 1960, 216 p.

6 John Russell Harper, La Peinture au Canada, des origines à nos jours, Sainte-Foy, Presses de l'Université Laval, 1966, 442 p.

7 John Russell Harper, Early Painters and Engravers in Canada, Toronto, University of Toronto Press, 1970, $376 \mathrm{p}$.

8 Mario Béland, dir., La Peinture au Québec: 1820-1850, Québec, Musée du Québec, 1991, 605 p.

9 Rosemarie L. Tovell, dir., Berczy, Ottawa, Musée des beaux-arts du Canada, 1991, 327 p.

10 Jim Burant et al., Un moment dans l'histoire, Ottawa, Archives nationales du Canada, 1991, 302 p.

11 David Karel, Dictionnaire des artistes de langue française en Amérique du Nord, Québec, Musée du Québec, Sainte-Foy, Presses de l'Université Laval, 1992, 962 p.

12 Certains extraits du présent article constituent des adaptations de ce mémoire. Anne-Elisabcth Vallée, L'Art de la miniature au BasCanada entre 1825 et 1850 : Gerome Fassio et ses contemporains, mémoire de maîtrise, Montréal, Université du Québec à Montréal, 2004, 270 p.

13 Dans la collection de BAC, les miniatures ont été acquises principalement en fonction du modèle représenté. Les conservateurs de la collcction ont souvent privilégié l'étude biographique des modèles plutôt que la recherche concernant les artistes ayant exćcuté ces œuvres.

14 Ces miniatures correspondent au portrait de la Femme de la famille Field de Cobourg, Ontario (1990-200-1) (fig. 4), aux œuvres de Gerald Sinclair Hayward (1845-1926) soit les portraits de Wilfrid Laurier (1982-213-1) et de Marie Madeleine de Verchères (1989497-1), ainsi qu'au portrait d'Alice Walker (1982-204-10).

15 I.es deux portraits réalisés dans les Maritimes sont celui de William Taylor (1934-308-1) et celui de Demasduwit (Mary March) (197714-1).

16 La miniature exécutée en Colombie-Britanniquc est l'œuvre de Louie Burrell représentant le Fïls de M. et Mme Crawley Ricardo de Victoria, Colombie Britannique (1983-89-2).

17 Juliette de Lavoye a exécuté les portraits miniatures de Jules Léger, gouverneur-général du Canada (1976-35-1), Auteure et peintre Sophy Louisa Elliott (1992-479-1), Madame Georgianne Chaussé, mère de l'artiste (1981-62-2), Monsieur J. Aldéric Raymond (1982-89-1) et Robert Choquette (1981-62-1).

18 Les portraits du Fils de M. et Mme Crawley Ricardo de Victoria, Colombie Britannique (1983-89-2) et d'une Femme non identifiée vers 1912-1916 (1983-89-3) sont des œuvres de I.ouie Burrcll.

19 Dans son mémoire intitulé Miniatures and Silhouettes in Montreal, 1760-1860, Roslyn M. Rosenfeld affirme: "The itinerant travelled through economic necessiry. No colonial centre, not even Boston or Philadelphia, could offer constant employment to miniaturists or silhouetrists. Montreal, though growing rapidly, had only nine thousand inhabitants at the turn of the nineteenth century. "Rosenfeld, op. cit., p. 13. Dans un même ordre d'idées, Robin Jaffcc Frank rapporte : "William Dunlap, [...], is known to posterity as both the "father of the American theatre" and the "American Vasari"; nonetheless, his long and varied career proved financially unrewarding. In his diary, the multi-faceted playwright, theatre manager, art historian, and portrait painter recounts a life spent wandering in pursuit of social contacts and commissions, time spent sleeping in rented rooms away from home ". Robin Jaffee Frank, Love and Loss. American Portrait and Mourning Miniatures, New Haven, Connecticut, Yale University Press, 2000, p. $169-72$.

20 En fair, le premier miniaturiste recensé, Monsieur de Milon, offre ses services dans la colonie par l'entremisc du journal The Montreal Gazette le 4 juin 1789. Après 1860, peu de miniaturistes étrangers séjournent au Canada. La demande pour les portraits miniatures y connaît une forte baisse depuis l'essor des procédés photographiques. Quelques miniaturistes d'origine canadienne poursuivent néanmoins leurs activités au pays. Pour en connaître davantage sur les premiers miniaturistes de passage dans la colonie, voir Rosenfeld, op. cit., 1981, 166 p.

21 Ibid., p. 13.

22 Par exemple, au mois de septembre 1819, l'artiste Cornelius Schroeder fait paraître une réclame dans le journal montréalais Canadian Courant dans le but d'informcr la population de son arrivée. Le miniaturiste reprend la même formule lorsqu'il visite la ville de Québec en août 1830. De même, en septembre 1829, un 
miniaturiste du nom de J. Wilson s'installe à Québec et annonce, par une publicité dans le journal The Quebec Mercury, l'ouverture d'une école de dessin en tout genre. Au mois de mai 1830, l'artistc publie dans The Montreal Gazette une annonce de ses services dc miniaturiste. Canadian Courant, 24 novembre 1819, p. 2; The Quebec Mercury, 12 septcmbre 1829, p. 471; The Montreal Gazette, 13 mai 1830, p. 2; The Quebec Mercury, 10 août 1830, p. 427.

23 Le groupe de miniaturistes et de silhouettistes connu sous le nom de "The Artists" propose dans le journal The Montreal Gazette du 5 mars 1833 d'exécuter des portraits de profil pour cinquante cents. Quant à l'artiste américain Henry Colton Shumway, selon H. W. French, il aurait parfois demandé jusqu'à trois cents dollars pour une seule miniature. The Montreal Gazette, 5 mars 1833, p. 3 ; H. W. French, Art and Artists in Connecticut, New York, Kennedy Graphics, Inc., 1970, p. 73-4.

24 Originaire des États-Unis, Deming débute sa carrière en exposant son travail à la National Academy of Design en 1833. Son premier séjour à Montréal est commenté dans The Montreal Transcript and Commercial Advertiser dans un article élogieux paru au mois de janvier 1843. Selon les informations inscrites à l'endos du portrait de John Fletcher, la miniaturiste séjourne encore ou à nouveau à Montréal durant l'été 1845. De même, l'inscription au verso de sa seconde œuvre connuc, le portrait du Révérend Micajah Townsend (collection particulière), révèle sa présence à Clarenceville dans les Cantons de l'Est au mois d'août 1846. L'année suivante, elle participe à la première exposition de la Montreal Gallery of Paintings débutant le 11 janvier 1847. Entre 1856 et 1876, Deming expose fréquemment à la National Academy of Design ainsi quà l'American Society of Painters of Water Colors. The Montreal Gazette, 15 janvier 1847, p. 2-3; The Montreal Transcript and Commercial Advertiser, 21 janvier 1843, p. 2, et 18 novembrc 1843, p. 3; The Pilot and Journal of Commerce, 29 janvier 1847, p. 2; George Cuthbert Groce et David H. Wallace, Dictionary of Artists in America, 1564-1860, New Haven, Conn., Yale University Press, 1964, c. 1957, p. 174.

25 Les atrributions des miniatures de BAC ont été réalisées par lcs conservateurs ainsi que par des chercheurs indépendants. Malhcureusement, les dossiers des œuvres conservées à BAC ne mentionnent pas l'identité des spécialistes ayant attribué les miniatures et ne fournissent souvent pas les justifications de ces attributions.

26 Natif de Middletown dans le Connecticut, Shumway loue unc chambre à l'Hôtel Rasco de Montréal au mois de novembre 1844. Sans doute confiant dans sa notoriété, Henry Colton Shumway fait paraître dans The Montreal Gazette une simple annonce précisant son activiré professionnelle et le numéro de sa chambre d'hôtcl. Ayant étudié à la National Academy of Design, le miniaturistc amorce sa carrière en 1829 dans la ville de New York. En 1838, il se rend à Washington pour y peindre, entre autres, la miniaturc d'après nature du Prince Napoléon (1822-1891). La qualité des œuvres de l'artiste, qui travaille surtout à New York, lui permet d'acquérir rapidement une excellente réputation dans le domaine, le rendant à même de hausser son tarif à trois cents dollars pour une seule miniature. Après 1860, l'importantc baisse de demande pour les portraits miniaturcs oblige l'artiste à se recycler, comme d'autres peintres, dans le lucratif marché de la retouche photographique. The Montreal Gazette, 21 novembre 1844, p. 2; French, op. cit., p. 73-4; Frederic Fairchild Sherman, "Henry Colton Shumway - American Miniaturist ", Art in America, vol. 31, juillet 1943, p. 155.

27 La composition plutôt traditionnelle du portrait, un buste inséré dans un médaillon ovale, ainsi que le cadrage très scrré de la tête du sujet rappellent les miniatures de John Frazee (1790-1852) et de son épousc, réalisées durant les années 1830. Ces deux portraits sont conservés à la Pennsylvania Academy of the Fine Arts à Philadelphie (46120761 et 46120762).

28 Le cas de James Duncan (1806-1881) constitue un parfait exemple de la multidisciplinarité permettant de demeurer en un seul endroit plus longtemps. En effet, durant sa carrière, James Duncan offrira ses services de miniaturiste, photographe, enseignant de dessin et peintre de paysage. (The Montreal Gazette, 27 octobre 1832, p. 1; La Minerve, 5 octobre 1843, p. 2; The Montreal Gazette, ler novembre 1856 , p. 2). De même, Robert Auchmuty Sproule (?1845) s'annonce d'abord en tant que miniaturiste puis il travaillera comme pcintre de paysage, cnseignant de dessin, ct enfin comme lithographc. (The Montreal Herald, 4 octobre 1826, p. 3; La Minerve, 17 mai 1832, p. 3; The Montreal Gazette, 8 mai 1834, p. 3).

29 Par exemple, dans sa première réclame dans les journaux de Québec, l'artiste Henry Daniel Thielcke (1788-1874) affirme qu'il se spécialise dans les portraits à l'huile et les miniatures. Nous lui connaissons quelques portrairs à l'huile mais, à ce jour, aucune miniaturc. Peut-être n'a-t-il pas poursuivi sa carric̀rc de miniaturiste dans la colonie? Quoi qu'il en soit, il est certain que jusqu'ici, l'étude de ses portraits de grand format n'a pas permis de lui attribuer des miniatures. The Quebec Mercury, 22 novembre 1832, p. 4.

30 Originairc d'Angleterre, Lockwood visite une première fois la ville de Québec au mois de juin 1833, alors qu'il est associć avec l'artiste John Popplewell. Il offre alors un large éventail de productions artistiqucs : miniatures sur ivoire et sur carton Bristol, mezzotinte, peinturc chinoise et japonaisc, silhouette, peinture sur velours ou satin, etc. A la recherche dc commandes, les deux artistes se rendent à Toronto l'année suivante, où ils exposent à la First Exhibition of the Sociery of Artists \& Amateurs of Toronto. Lockwood se trouvera à nouveau à Toronto en mars 1841. Trois ans plus tard, il exécute le portrait du gouverneur général Sir Charles Metcalfe. I a même année, l'arriste s'installe à Québcc et offre ses services de miniaturiste. Il résidera principalement dans cette ville entre 1845 ct 1848 . Lockwood demeure au Québec jusqu'à son décès en février 1866. The Quebec Mercury, $1^{\text {er }}$ juin 1833, p. 3; 26 octobre 1844 , p. 3 ; 31 octobrc 1844, p. 2; 16 novembre 1844 , p. 3; 30 novembre 1844 , p. 3; 4 septembre 1845 , p. 3; 25 juin 1846, p. 3; 25 juin 1846, p. 3; Le Courrier du Canada, 23 février 1857, p. 3. Mackay's Montreal Directory for 1861, Montréal, J. Lovell, p. 136. Le Journal de Québec, 8 février 1866, p. 3. Harper, op. cit., 1970, p. 199.

31 La présence d'une carte du commerce montréalais porte à croire que le portrait a tout simplement été recadré entre 1906 et 1910. 
période durant laquelle The Art Emporium fait affaire à l'adresse indiquée. Notons que cette miniature provient de la collection offerte aux Archives nationales du Canada en 1959 par Sir Donald Alexander Smith, baron de Strathcona. L'histoire de la garde de l'œuvre ne nous est pas parvenue. Lovell's Montreal Directory, Montréal, Lovell, 1905-1910.

32 Nous urilisons le terme d'" amateur " non pas dans un sens péjoratif, mais plutôt par opposition aux artistes professionnels, en faisant référence aux personnes qui pratiquent leur art simplement par plaisir et qui ne sont pas payées pour leurs œuvres.

33 Le Journal du Noviciat de l'Hôpital général de Québec (extraits communiqués à Gérard Morisset par sœur Saint-Alphonse en janvier 1935), 5 février 1842, p. 47. Monastère des Ursulines (Québec), Les Ursulines de Québec depuis leur établissement jusqu'à nos jours, Québec, C. 1)arveau, 1863-1866, p. 674-75.

34 "Distribution solennelle des prix aux élèves des Dames Ursulines de Québec ", Le Journal de Québec, 18 juillet 1846, p. 1.

35 Béland, dir., op. cit., p. 506.

36 Ibid., p. 266.

37 Harper, op. cit., p. 39; Karcl, op. cit., p. 107.

38 Deux artistes sont en exil avec Nelson : Robert-Shore-Milnes Bouchette et Jean-Joseph Girouard (1794-1855). L'analyse stylistique de la miniature de Nelson ne correspond pas aux autres ouvrages du même genre réalisés par Girouard.

39 G. M. Story, "Demasduwit (Shendoreth, Waunarhoake, Mary March) ", Dictionnaire biographique $d u$ Canada, Sainte-Foy, I.es Presses de l'Université Laval, vol. V, 1983, p. 267-68. Susan North, "Henrietta Martha Hamilon (c. 1780-1857) ", Un moment dans l'histoire, op. cit., p. 168-70.

40 Rosenfeld, op. cit.

41 Daphne Foskett, A Dictionary of British Miniature Painters, Londres, Faber and Faber, 1972, 2 volumes. Frank, op. cit., 358 p. Rosalind M. Pepall, et Elizabeth Kennel, Portraits en miniature de la collection du Musée des beaux-arts de Montréal, Montréal, Muséc des beaux-arts de Moncréal, 1989, $91 \mathrm{p}$.

42 Foskett, op. cit., vol. 1, p. 126; Frank, op. cit., p. 15-34; Pepall et Kennel, ibid., p. 18.; Rosenfeld, op. cit., p. 31-4.

43 Foskett, op. cit., vol. 1, p. 127; Frank, op. cit., p. 229-74; Pepall et Kenncl, op. cit., p. 20; Rosenfeld, op. cit., p. 41-9.

44 Frank, op. cit.; Pepall et Kennel, op. cit.

45 Frank, op. cit., p. 277-303; Rosenfeld, op. cit., p. 56-8.

46 Frank, op. cit., p. 280.

47 Le négatif sur verre au collodion humide intitulé Wilfrid Laurier, Ottawa, Ont., vers 1890 (MP-25.932), ct provenant du Studio Topley, est conservé au Musée McCord d'histoire canadienne. L'une des estampes produites à partir de ce portrait appartient au Musće de la civilisation, Québec (1993.17163).

48 Arthur Doughty, A Daughter of New France : Being a Story of the Life and Times of Magdelaine de Verchères (1665-1692), Otcawa, Mortimer Press, 1916, 166 p.

49 Susan Stewart, On Longing. Narratives of the Miniature, the Gigantic, the Souvenir, the Collection, Baltimore et Londres, The Johns Hopkins University Press, 1984, p. 54-68.

50 Pour des exemples de ces miniatures offertes ou exécutées à des moments marquants de la vie du commanditaire ou du modèle, voir Frank, op. cit., p. 34-5, 39) et 140.

51 Par exemple, au Bas-Canada, le taux de mortalité est très élcvé chez les nouveaux-nés, attcignant près de $25 \%$ entre 1821 ct 1871 . Jcan-Claude Robert, Atlas historique de Montréal, Montréal, Art Global, Libre Expression, 1994, p. 94.

52 Le Musée royal de l'Ontario possède un portrait miniature d'Angélique de Lotbinière (977.234.1) exécuté de mémoire vers 1818 par Louise-Amćlie Panet (1789-1862), qui connaissait le jeune modèle. Tovell, dir., op. cit., p. 294.

53 Davida Tenenbaum Deutsch, "Jewelry for Mourning, Love, and Fancy, 1770-1830", The Magazine Antiques, vol. CLV, avril 1999, p. 568.

54 I.e Musée David M. Stewart conserve un exemple de cette pratique. À l'endos de la miniature représentant Luc de La Corne, unc Allégorie représentant Marie-Anne-Marguerite Boucher de Boucherville el sa fille Marie-Marguerite de La Corne devant le monument funéraire de Luc de La Corne (1972.479) a été peinte à l'aquarelle sur ivoire. Ce portrait de La Corne ainsi que l'image de deuil au verso ont probablement été exécutés par un ou deux artistes européens.

55 Fosketr, op. cit., vol. 1, p. 124-25.

56 lbid., p. 126.

57 Marcia Pointon, "Surrounded with Brilliants" : Miniature Portraits in Eighteenth-Century England ", The Art Bulletin, vol. I.XXXIII, mars 2001, p. 48.

58 Concernant les types d'encadrements, consulter Foskett, op. cit., p. 123-27, et Pointon, op. cit., p. 48-71.

59 Foskett affirmc: "In the seventeenth century the frames became plainer, and were usually silver or gold with a scroll over the top edge. [...] During the early part of the eighteenth century when the miniatures were smaller in size, most of the frames wcre plain silver or gold, made as lockets or brooches". Foskett, op. cit., p. 127.

60 Eva Major-Marothy, "Basilique-Benjamin Trotrier Desrivières Beaubien ", dans Mario Béland, dir., op. cit., p. 181-82.

61 Heinz K. et Bridget A. Henisch, The Photographic Experience 18391914. Images and Attitudes, Philadelphie, The Pennsylvania State Universicy Press, 1994, p. 140.

62 Jean-Michel Ribettes, Fétiches ơ Fétichismes, catalogue d'exposition, Paris, Blanche, Passage de Rer', 1999, p. XXVII-XI.V.

63 Ibid., p. XXV.

64 Ibid., p. XXIII ct XXIV.

65 Ibid., p. XXXI.

66 F́douard Pommier, Théories du portrait. De la Renaissance aux Lumières, Paris, Gallimard, 1998, p. 265.

67 J. Wilson, "To a miniature picture (of an adopted Sister) ", The Montreal Monthly Magazine, mars 1831, p. 15. Le poème se poursuit:

With kindred kindness, glowing in her breast,

Picture of innocence, untaught by cunning art,

Features expressive of a tender heart,

A mind unbias'd, may thy breast concain,

And there may every social virtue reign,

Heaven blast the wretch, who with perfidious art, 
Of feigned affection, would ensnare thy heart,

Or dim the lustre of that lovely eye,

Or make that tender bosom heave a sigh,

At thy complaint thy brother's martial arm,

Is ready to protect thee, free from harm,

If wronged revenge thee, if man the offender is,

My sword shall search the traitor's heart, or his

My bosom shall reccive, 'tis well prepared,

A smile from thec in death, a good reward.

But all prospitious heaven, her youth protect,

Guard her through life, and may she ne'r forget

To ask thy powerful protection,

And I remain with warm affection,

Her loving Brother, J. W.

68 A ce propos, consulter Deutsch, op. cit., p. 566-75, et Irene Guggenheim Navarro, "Hairwork of the Nineteenth Century ", The Magazine Antiques, vol. CLIX, mars 2001, p. 484-93.
69 Dans la collection de BAC, aucune miniature identifiée avec certitude comme étant de production canadienne ne présente d'" ouvrages en cheveux " à l'endos du médaillon. Nous n'avons d'ailleurs aucune preuve nous permettant de confirmer ou d'infirmer la présence d' " artistes en cheveux " au Canada. Cependant, certaines miniatures canadiennes conservées par d'autres institurions muséales ou par des collectionneurs privés comportent de tels ouvrages à l'endos du médaillon. Par exemple, la miniature de Pierre-Amable De Bonne, cuvre de William Berczy appartenant à un descendant de la famille Marcoux (la famille de l'épouse du modèle), comporte au verso de son médaillon un motif de gerbe formé de cheveux et de fleurs. Navarro, ibid.; Tovell, dir., op. cit., p. 215.

70 Dans son article, Navarro remarque que l'utilisation de cheveux en tant que pigment, popularisée durant le XVIIIe siècle, décline après 1830. Navarro, op. cit., p. 489. 